\title{
Effects of confinement on anomalies and phase transitions of core-softened fluids
}

Cite as: J. Chem. Phys. 142, 134502 (2015); https://doi.org/10.1063/1.4916563

Submitted: 12 February 2015 . Accepted: 18 March 2015 . Published Online: 02 April 2015

Leandro B. Krott, José Rafael Bordin, Ney M. Barraz (D), and Marcia C. Barbosa

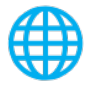

\section{ARTICLES YOU MAY BE INTERESTED IN}

Confinement effects on the liquid-liquid phase transition and anomalous properties of a monatomic water-like liquid

The Journal of Chemical Physics 143, 244503 (2015); https://doi.org/10.1063/1.4937486

Distinct dynamical and structural properties of a core-softened fluid when confined between fluctuating and fixed walls

The Journal of Chemical Physics 139, 154502 (2013); https://doi.org/10.1063/1.4824860

High pressure induced phase transition and superdiffusion in anomalous fluid confined in flexible nanopores

The Journal of Chemical Physics 141, 144502 (2014); https://doi.org/10.1063/1.4897956

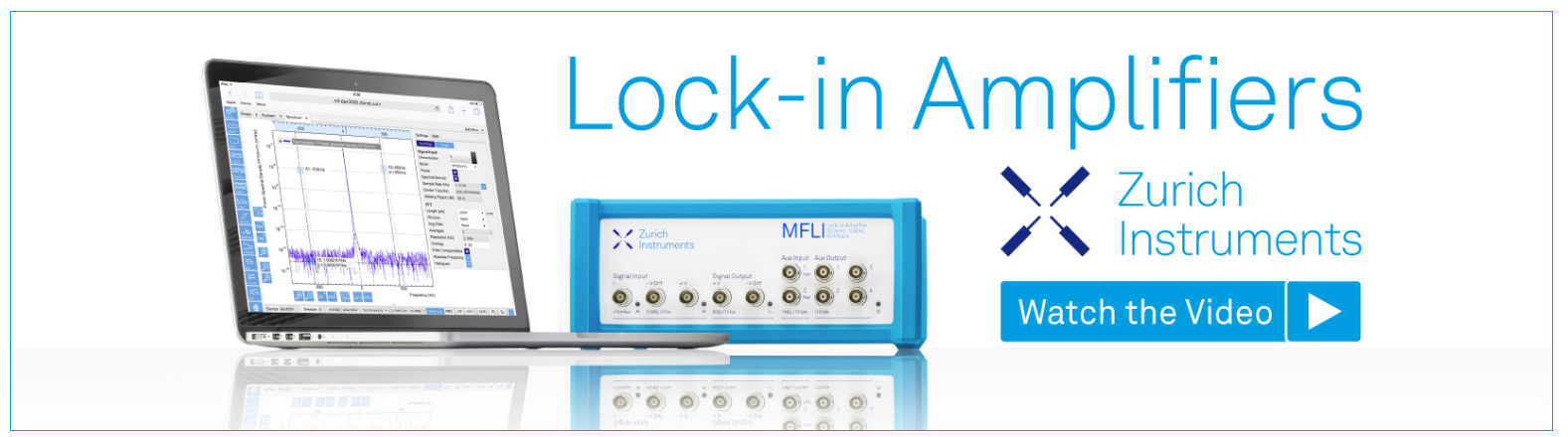




\title{
Effects of confinement on anomalies and phase transitions of core-softened fluids
}

\author{
Leandro B. Krott, ${ }^{1, a)}$ José Rafael Bordin, ${ }^{2, b)}$ Ney M. Barraz, Jr., \\ and Marcia C. Barbosa $\left.{ }^{1, d}\right)$ \\ ${ }^{1}$ Instituto de Física, Universidade Federal do Rio Grande do Sul, Caixa Postal 15051, CEP 91501-970, \\ Porto Alegre, RS, Brazil \\ ${ }^{2}$ Campus Caçapava do Sul, Universidade Federal do Pampa, Av. Pedro Anunciação, s/n, CEP 96570-000, \\ Caçapava do Sul, RS, Brazil \\ ${ }^{3}$ Campus Cerro Largo, Universidade Federal da Fronteira Sul, Av. Jacob Reinaldo Haupenthal, \\ 1580. CEP 97900-000, Cerro Largo, RS, Brazil
}

(Received 12 February 2015; accepted 18 March 2015; published online 2 April 2015)

\begin{abstract}
We use molecular dynamics simulations to study how the confinement affects the dynamic, thermodynamic, and structural properties of a confined anomalous fluid. The fluid is modeled using an effective pair potential derived from the ST4 atomistic model for water. This system exhibits density, structural, and dynamical anomalies, and the vapor-liquid and liquid-liquid critical points similar to the quantities observed in bulk water. The confinement is modeled both by smooth and structured walls. The temperatures of extreme density and diffusion for the confined fluid show a shift to lower values while the pressures move to higher amounts for both smooth and structured confinements. In the case of smooth walls, the critical points and the limit between fluid and amorphous phases show a non-monotonic change in the temperatures and pressures when the nanopore size is increase. In the case of structured walls, the pressures and temperatures of the critical points varies monotonically with the pore size. Our results are explained on basis of the competition between the different length scales of the fluid and the wall-fluid interaction. (C) 2015 AIP Publishing LLC. [http://dx.doi.org/10.1063/1.4916563]
\end{abstract}

\section{INTRODUCTION}

Water is an important material in industry, technology, and biological processes due to its unusual properties. Water unusual properties comprise many anomalous behavior, with 70 known anomalies, ${ }^{1}$ like the maximum value of its density in $T=4{ }^{\circ} \mathrm{C}$ at room pressure, and the increase of the diffusion as the system is compressed. ${ }^{2-4}$ These anomalies have been explained in terms of the formation of hydrogen bond network. The water molecules form open and compact (bonded and nonbonded) clusters of tetramers. From the competition between these structures the anomalies arise.

As a natural consequence of the polymorphism of water clusters, the pressure-temperature phase diagram of water is very complex. At low temperatures, water shows a coexistence of two amorphous phases: a low density amorphous and a high density amorphous. For higher temperatures, these two amorphous phases might lead to the appearance of two liquid phases, separated by a first order phase transition line ended in a liquid-liquid critical point $(L L C P)$. Whereas, homogeneous nucleation occurs in this region, that is called no man's land, and because of that, it is an incredible hard task to do experimental measures of liquid water in bulk systems in this region. Theoretically, the existence of these two liquid phases was evidenced in the atomistic ST4 model by Poole and

\footnotetext{
a)leandro.krott@ufrgs.br

b)josebordin@unipampa.edu.br

c) ney.barraz@uffs.edu.br

d) marciabarbosa@ufrgs.br
}

co-authors ${ }^{5}$ and confirmed in recent simulations. ${ }^{6,7}$ As well, new experiments suggest the coexistence of a high-density and low-density liquid phase of water. ${ }^{8} \mathrm{~A} L L C P$ was also predicted for others atomistic models of water, ${ }^{9-14}$ and in models for phosphorus, ${ }^{15}$ silica, ${ }^{16,17}$ silicon, ${ }^{18}$ carbon, ${ }^{19}$ hydrogen, ${ }^{20}$ and colloidal systems. ${ }^{21}$ On the other hand, recent studies suggests that the $L L C P$ can be an open trend on supercooled water and other materials. ${ }^{22-24}$ In this way, there is still several open questions about the $L L C P$.

As an attempt to avoid the crystallization of water in the no man's land, experiments with nanoconfined water have been performed recently. ${ }^{25-27}$ The presence of a confining structure changes the number of hydrogen bonds, avoiding the nucleation. Some experiments of water confined in nanopores, performed by $x$-ray and neutron scattering, show that liquid states persist down to temperatures much lower than in bulk. ${ }^{28-30}$ The nanopores' size has important influence in the crystallization of the system, ${ }^{28,30-33}$ and hydrophilic and hydrophobic nanopores can lead to distinct results as well. ${ }^{29,34}$

Classical atomistic models for water are important tools to understand its properties. On the other hand, coarse-grained models arise as an interesting tool to see the universal mechanisms that lead to anomalous waterlike properties. Coarsegrained models may reproduce diffusion and density anomalies and can be modeled by core-softened (CS) potentials with two length scales, which can be constructed using a shoulder or a ramp potential. ${ }^{35-40}$ These coarse-grained models for anomalous fluids are able to capture the bulk waterlike anomalies and averaged properties in the confined materials. When 
confined by fixed hydrophilic plates, the fluid-wall interaction can induce solidification and shift the anomalous properties to higher temperatures, while hydrophobic nanopores lead the system to remain in liquid state and shift the waterlike anomalies to lower temperatures in relation to bulk. ${ }^{41,42}$ Whereas, when the nanopore has at least one degree of freedom, given by the mobility of the plates in $z$ direction, the anomalous behavior of the fluid disappears and distinct phase transitions are observed. ${ }^{43-46} \mathrm{CS}$ fluids confined in nanotubes also present interesting findings, similar to obtained in atomistic models for water, as the increase in diffusion coefficient and flux for narrow nanotubes associated to a layer to single-file transition and a discontinuity in the enhancement flow factor. ${ }^{47-49}$ The drawback of these core-softened potentials is that due to the simplicity of the two length scales, they are not capable to reproduce the effects related to the third coordination shell of the anomalous fluid what might be relevant under confinement. $^{50}$

In addition to the relevance of the detail structure of the liquid, the structure of the confining system is also relevant since biological and physical materials do not exhibit the smoothness and regularity of the flat walls and tubes employed in the simulations. This naturally raises the question of what is the role played by the structure of the liquid and of the interface in the thermodynamic, dynamic, and structural behaviors of confined systems. Recent simulations have shown that the hydrophobic or hydrophilic behavior of the confining surfaces is governed by the interfacial free energy, which strongly depends on the surface structure. ${ }^{51}$ Even thought these simulations do not observe important differences in the diffusion of the systems confined between smooth and rough walls, ${ }^{52}$ they show that the adsorption behavior and the solvation pressure are significantly affected by the roughness of the confining surface $^{53}$ and that different liquid and solid phases that exist in the smooth confined are not present in the rough case. ${ }^{44}$

In this work, we address the question of which are the effects of the roughness of the nanopore wall in the physical properties of an anomalous fluid. Our analysis is done in the framework of an effective model that incorporates not only the two length scales traditionally present in the CS potentials but also additional length scales representing the third coordination shell of the fluid. Our goal is also to understand the effect of the structure of the liquid in the thermodynamic, dynamic, and structural properties of a fluid confined in a nanopore.

The paper is organized as follows: in Sec. II, we introduce the model and the methods and simulation details are described; the results are given in Sec. III; and conclusions are presented in Sec. IV.

\section{THE MODEL AND SIMULATION DETAILS}

In this paper, all physical quantities are computed in the standard LJ units, ${ }^{54}$

$$
r^{*} \equiv \frac{r}{r_{0}}, \quad \rho^{*} \equiv \rho r_{0}^{3}, \quad \text { and } \quad t^{*} \equiv t\left(\frac{\gamma}{m r_{0}^{2}}\right)^{1 / 2}
$$

for distance, density of particles, and time, respectively, and

$$
p^{*} \equiv \frac{p r_{0}^{3}}{\gamma}, \quad U^{*} \equiv \frac{U}{\gamma}, \quad \text { and } \quad T^{*} \equiv \frac{k_{B} T}{\gamma}
$$

for the pressure, energy, and temperature, respectively, where $r_{0}=2.869 \AA$ is the distance parameter, $\gamma=0.30 \mathrm{kcal} / \mathrm{mol}$ the energy parameter, and $m$ the mass of each particle. Since all physical quantities are defined in reduced LJ units, the ${ }^{*}$ is omitted, in order to simplify the discussion.

The fluid is composed by $N$ spherical particles of diameter $\sigma=1.47$ and mass $m$ confined between two parallel and fixed plates. We have studied two kinds of nanopores: with smooth and structured walls. Smooth plates are modeled by force fields and do not have structure, interacting continuously with the fluid. Structured plates are formed by spherical particles in a square lattice with punctual interactions. A schematic depiction for the systems with (a) smooth and (b) structured plates is shown in Fig. 1.

The particles of the fluid interact through the isotropic effective potential ${ }^{55}$ given by

$$
\frac{U(r)}{\epsilon}=\left[\left(\frac{\sigma}{r}\right)^{a}-\left(\frac{\sigma}{r}\right)^{b}\right]+\sum_{j=1}^{4} h_{j} \exp \left[-\left(\frac{r-c_{j}}{w_{j}}\right)^{2}\right],
$$

with the parameters given in the Table I. Fig. 2 shows the potential versus distance in dimensionless units. In this work, we use $\epsilon / \gamma=0.02$.

This effective potential was derived from the OrnsteinZernike and integral equations applied to the oxygen-oxygen radial distribution function of the atomistic model ST4. ${ }^{58} \mathrm{At}$ short distances, it shows two characteristic length scales: one at $r_{1} \approx 1.1$ and another at $r_{2} \approx 1.6$, as in the usual coarse grained potentials proposed to model the anomalous liquids. In addition, a third length scale at $r_{3} \approx 2.2$ is also present. Since the derivation of the potential was based on the oxygen radial distribution function, these length scales represent the oxygenoxygen distances related to the relevant coordination shells in the liquid. The bulk system exhibits waterlike anomalies, and the liquid-gas and liquid-liquid critical points predicted for water. ${ }^{55}$

In the confined system, the particles of the fluid interact with the wall by the Weeks-Chandler-Andersen (WCA) potential,

$$
U(r)=\left\{\begin{array}{l}
U_{\mathrm{LJ}}(r)-U_{\mathrm{LJ}}\left(r_{c w}\right), \quad r \leq r_{c w}, \\
0, \quad r>r_{c w},
\end{array},\right.
$$

where $U_{L J}(r)$ is the standard 12-6 LJ potential. ${ }^{54}$ The cutoff distance is $r_{c w}=2^{1 / 6}$. For smooth plates, the interaction occurs just in $z$ direction and the potential is written as $U_{L J}(z)$.
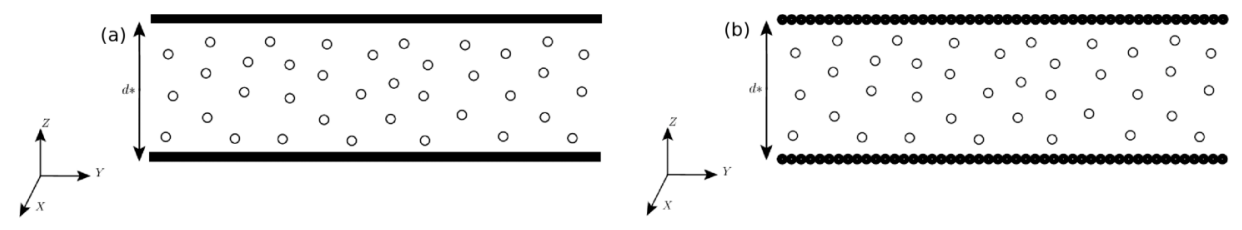

FIG. 1. Schematic depiction of the particles confined between (a) smooth and (b) structured plates. 
TABLE I. Parameters of the particle-particle potentials in units of $\AA$ and $\mathrm{kcal} / \mathrm{mol}$.

\begin{tabular}{lccccccc}
\hline \hline Parameters & Values & Parameters & Values & Parameters & Values & Parameters & Values \\
\hline$a$ & 9.065 & $w_{1}$ & 0.253 & $h_{1}$ & 0.5685 & $c_{1}$ & 2.849 \\
$b$ & 4.044 & $w_{2}$ & 1.767 & $h_{2}$ & 3.626 & $c_{2}$ & 1.514 \\
$\epsilon$ & 0.006 & $w_{3}$ & 2.363 & $h_{3}$ & -0.451 & $c_{3}$ & 4.569 \\
$\sigma_{p}$ & 4.218 & $w_{4}$ & 0.614 & $h_{4}$ & 0.230 & $c_{4}$ & 5.518 \\
\hline \hline
\end{tabular}

The dynamic, thermodynamic, and structural properties of the fluid was studied using molecular dynamics simulation in the NVT ensemble. The Nose-Hoover thermostat ${ }^{56,57}$ was used to fix the temperature, with a coupling parameter $Q=2$. The interaction potential between particles, Eq. (3), has a cutoff radius $r_{c}=3.5$.

The fluid was confined by two different kinds of parallel walls: smooth and structured. The plates are fixed and are located each one at $z=0$ and $z=d$. The smooth plates are modeled by force fields in $z$ direction and have no structure. The interaction between smooth plates and the fluid was done using the WCA potential, like shown in Eq. (4), but considering just the $z$ component. The structured plates are constructed by placing spherical particles of effective diameter $\sigma$ in a square lattice of area $L^{2}$. The fluid-wall interaction also is given by a WCA potential.

In $z$ direction, the space occupied for the fluid was limited by the confining plates. Due the excluded volume between the fluid near to the plates, the distance $d$ between them need to be corrected to an effective distance $d_{e}$, which can be approach by $d_{e} \approx d-\sigma \cdot{ }^{59}$ Consequently, the effective density will be $\rho=N /\left(d_{e} L^{2}\right)$.

Systems with plate separations $d=2.5,4.2,5.2$, and 8.0 were analyzed. Several densities and temperatures were simulated to obtain the full phase diagrams for each case. For systems with $d=2.5,4.2$, and 5.2, $N=507$ particles were employed, while for $d=8.0, N=546$ particles were used. Two different initial configurations of the systems were simulated: solid and liquid states. Using different initial configurations allows us to identify precisely the final state of the system, avoiding metastability. The equilibrium state was reached after $4 \times 10^{5}$ steps, followed by $8 \times 10^{5}$ simulation run. We used a time step $\delta t=0.001$, in reduced units, and all the physical quantities were get with 50 uncorrelated samples. To check the stability of the systems, we verify the energy as a function of time and the perpendicular pressure and parallel pressure as a function of density.

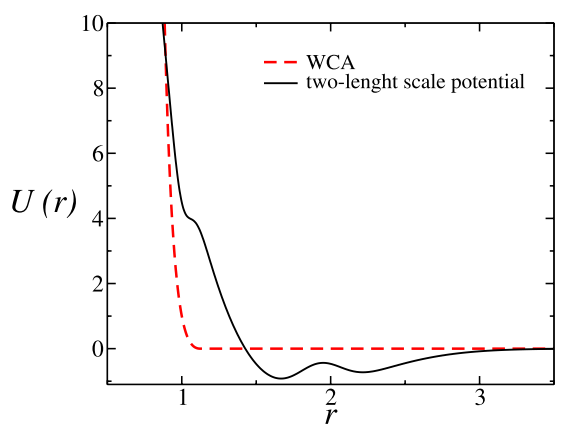

FIG. 2. Particle-particle interaction potential (black solid line) and particleplate interaction potential (red dashed line).
Since the fluid is confined in the $z$ direction, the thermodynamic averages were calculated in components parallel and perpendicular to the plates. ${ }^{60}$ The systems have periodic boundary conditions in $x$ and $y$ directions and they are extensive just in area and not in the distance between the plates.

The parallel pressure was calculated using the Virial expression for the $x$ and $y$ directions, ${ }^{59}$

$$
P_{\|}=\rho k_{B} T+\frac{1}{2 V}\left\langle\mathcal{V}_{\|}\right\rangle,
$$

where $\mathcal{V}_{\|}$is given by

$$
\mathcal{V}_{\|}=-\sum_{i=1} \sum_{j>i} \frac{x_{i j}^{2}+y_{i j}^{2}}{r_{i j}}\left(\frac{\partial U(r)}{\partial r}\right)_{r=r_{i j}} .
$$

The lateral diffusion coefficient, $D_{\|}$, was calculated using the mean square displacement (MSD), related from Einstein relation,

$$
D_{\|}=\lim _{\tau \rightarrow \infty} \frac{\left\langle\Delta r_{\|}(\tau)^{2}\right\rangle}{4 \tau},
$$

where $r_{\|}=\left(x^{2}+y^{2}\right)^{1 / 2}$ is the parallel distance of the particles.

The structure of the system was studied considering the lateral radial distribution function, $g_{\|}\left(r_{\|}\right)$, calculated in specific slabs between the plates. The definition of the $g_{\|}\left(r_{\|}\right)$is usually given by

$g_{\|}\left(r_{\|}\right) \equiv \frac{1}{\rho^{2} V} \sum_{i \neq j} \delta\left(r-r_{i j}\right)\left[\theta\left(\left|z_{i}-z_{j}\right|\right)-\theta\left(\left|z_{i}-z_{j}\right|-\delta z\right)\right]$.

The $\theta(x)$ is the Heaviside function and it restricts the sum of particle pairs in the same slab of thickness $\delta z=\sigma$. The $g_{\|}\left(r_{\|}\right)$is proportional to the probability of finding a particle at a distance $r_{\|}$from a referent particle.

\section{RESULTS}

\section{A. Thermodynamic, dynamic, and structural behavior smooth plates}

A schematic depiction of the system confined by smooth plates is shown in Figure 1(a). First, the effect of the structure of the liquid when confined by an uniform field is checked. Fig. 3 illustrates the transversal density profiles for plates separated at (a) $d=2.5$, (b) $d=4.2$, (c) $d=5.2$, and (d) $d$ $=8.0$ at $T=0.80$ and several densities. In all cases the system form layers, however the number of layers is dependent on the degree of confinement and of the density of the systems, what is consistent with results for atomistic such as $\mathrm{SPC} / \mathrm{E}^{61}$ and coarse grained approximations with three body terms as $\mathrm{mW}^{62}$ model. 

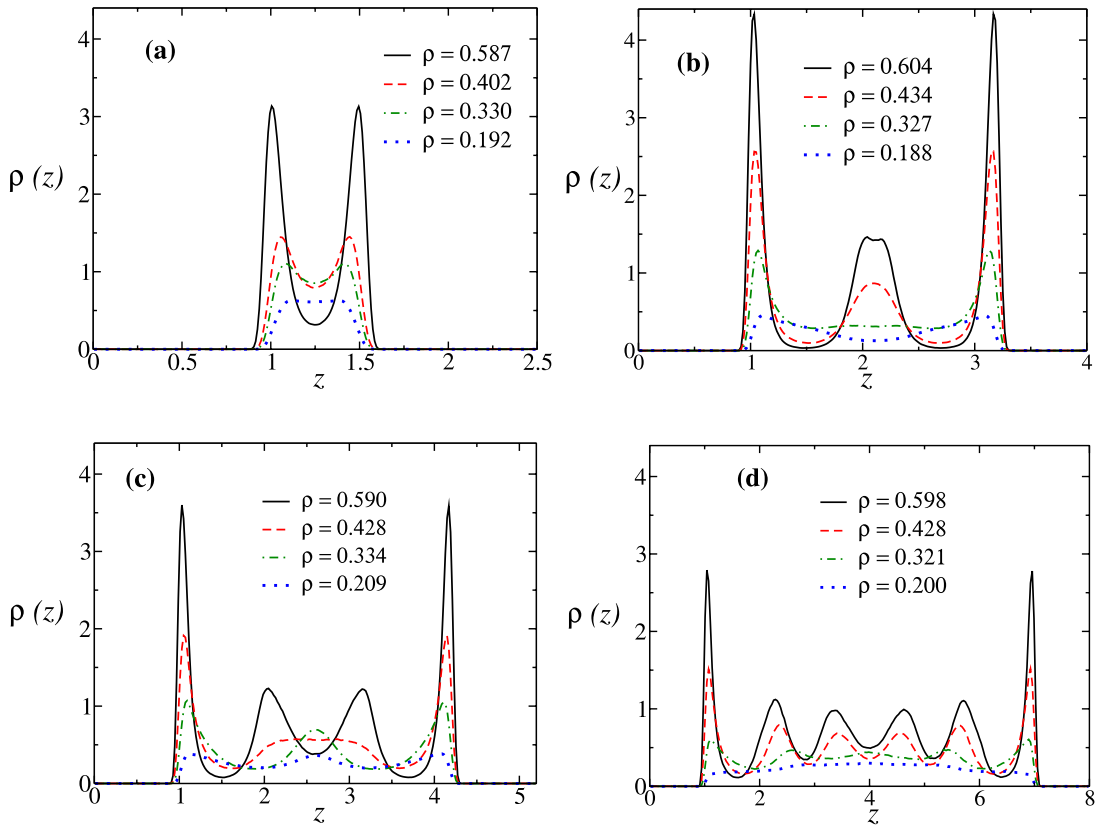

FIG. 3. Transversal density profiles for systems confined by smooth plates with $T=0.80$ and different densities at (a) $d=2.5$, (b) $d=4.2$, (c) $d=5.2$, and (d) $d=8.0$.
For higher degrees of confinement, $d=2.5$, the fluid is structured in one or two layers, depending on the density of the system. Fig. 3(a) shows that two layers are observed for high densities $(\rho=0.587)$, while one layer occurs for low densities $(\rho=0.192)$. The mechanism for the presence of different structures goes as follows. For low densities, the wall does not induce correlations and layering at the $z$ direction therefore one layer or bulk structure is formed. As the density becomes higher, the competition between particle-particle and wallparticle interactions leads to the formation of layers. There are two typical separations for the layers. The first is $r_{3}-r_{1}$ $\approx 1.1$, which corresponds to the minimum of energy, and the second one is $r_{2}-r_{1} \approx 0.5$, which is the second lowest energy potential. Since the plate separation is $d=2.5$, the confinement do not allow the fluid particles to remain in the first distance (minimum energy), and the layers separation is then equal to the second typical separation.

For other degrees of confinement, $d=4.2,5.2,8.0$, the same competition between wall-particle and particle-particle interactions appears as shown in Fig. 3. For low densities, an uniform distribution with just one layer appears, and as the density increases, two, three, four, or even five layers are present. However, since in this case the plates are further apart, the interlayer distance is $r_{3}-r_{1} \approx 1.1$ that corresponds to the distance between the shoulder length scale and the third coordination shell in Figure 2.

The diffusion anomaly observed in liquid water is characterized by the increase of the diffusion coefficient of the fluid when the pressure, or density, increases. For normal fluids, this coefficient decreases when the fluid is compressed. Fig. 4
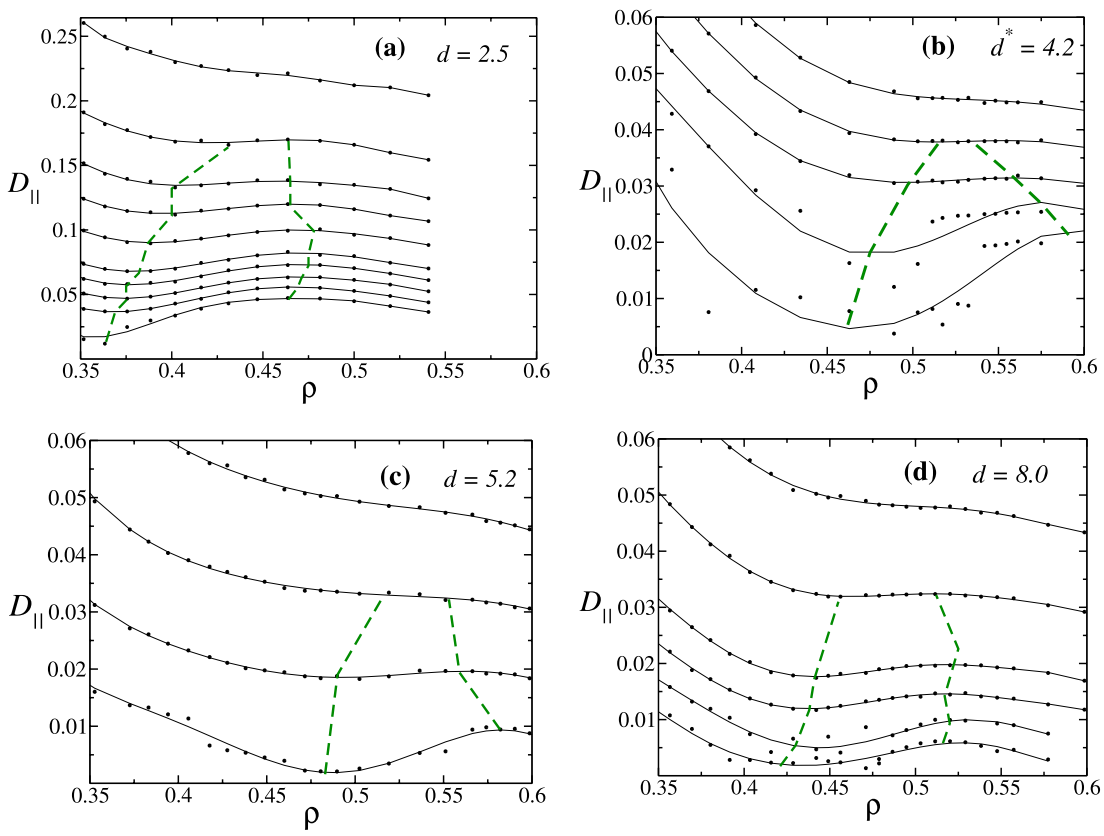

FIG. 4. Diffusion coefficient as a function of density for (a) $d=2.5$ and isotherms $0.60,0.65,0.70,0.75,0.80$, $0.90,1.00,1.10,1.25$, and 1.50 ; (b) $d=4.2$ and isotherms $0.60,0.65$, $0.70,0.75$, and 0.80 ; (c) $d=5.2$ and isotherms $0.50,0.60,0.70$, and 0.80 ; and (d) $d=8.0$ and isotherms 0.45 , $0.50,0.55,0.60,0.70$, and 0.80 . The dots are the simulated data and the black solid lines are polynomial fits. The dashed green lines bound the region where the diffusions are anomalous. 

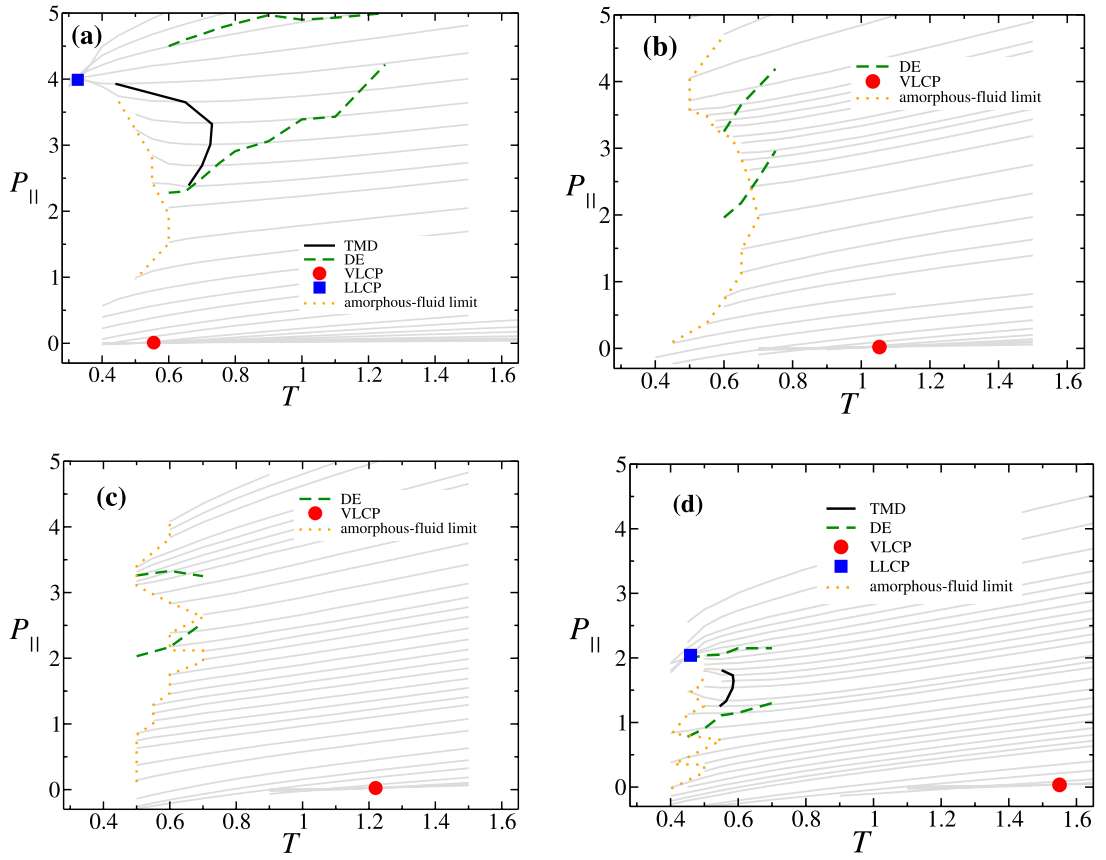

FIG. 5. Parallel pressure versus temperature phase diagram for systems with smooth plates separated by distances (a) $d=2.5$, (b) $d=4.2$, (c) $d$ $=5.2$, and (d) $d=8.0$. The thin lines represent different isochores. shows the lateral diffusion coefficient $\left(D_{\|}\right)$as a function of density of the system for (a) $d=2.5$, (b) $d=4.2$, (c) $d=5.2$, and (d) $d=8.0$. The range in temperature and density for which the anomaly in diffusion is the same for the distances $d=4.2,5.2,8.0$ but is different at $d=2.5$. These two behaviors, one at $d=2.5$ and another at larger distances might be related with the different length scales involved in the close and larger distances as observed in Figure 3.

In addition to the anomalous dynamic properties of the confined liquid, the thermodynamic and phase space were also explored. The system with $d=2.5$ illustrated in Fig. 5(a) shows the presence of a Temperature of Maximum Density, $T M D$, as a solid line, a vapor phase, and two liquid phases. This system, therefore, exhibits two stable critical points: a vapor-liquid critical point, VLCP, at $P_{c}=0.08$ and $T_{c}=0.55$ (red circle) and a $L L C P$ at $P_{c}=4.0$ and $T_{c}=0.3$ (blue square).
In the bulk system, the $V L C P$ occurs at $P_{c}=0.078$ and $T_{c}$ $=1.98$ while the $L L C P$ appears at $P_{c}=1.86$ and $T_{c}=0.48$. The comparison between the confined and the bulk systems indicates that the VLCP was shifted to lower temperatures but did not present significantly changes in pressure. Meanwhile, the $L L C P$ is shifted to lower temperatures and higher pressures in relation to bulk, what is in agreement with results obtained for theoretical models involving anomalous fluids ${ }^{63}$ and TIP4P water. ${ }^{64}$ The dashed lines in Fig. 5(a) represent the diffusion extremes and the pointed line indicates the limit between amorphous and fluid phases. The shifting of the critical point to lower temperatures can be assumed as a natural effect of the confinement, since the nanopore walls increase the entropy of the free energy of the system, favoring the disordered fluid phase. The increase in the pressure for the appearance of the $L L C P$ is the result of the layering imposed by the walls. The
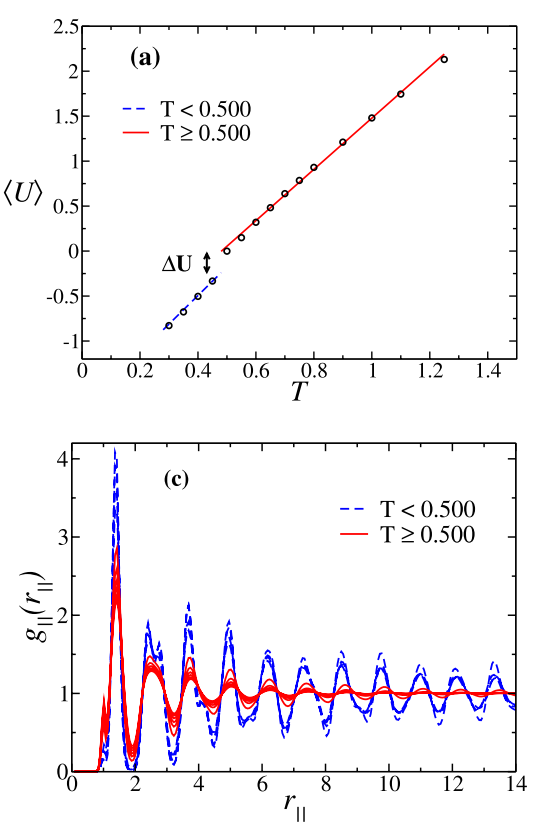
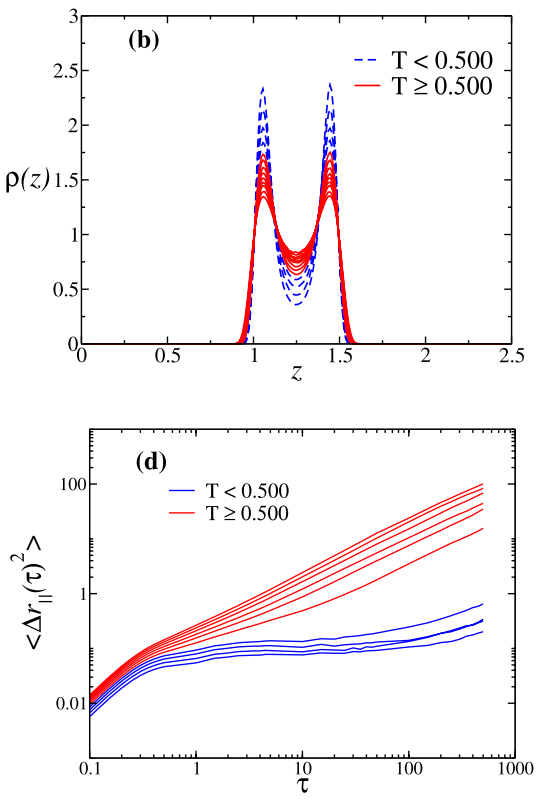

FIG. 6. System with plates separated at $d=2.5$ and density $\rho=0.402$. In (a), the mean potential energy as a function of temperature; in (b), the transversal density profile; in (c), the lateral radial distribution function $\left(g_{\|}\left(r_{\|}\right)\right)$for the contact layer; and in (d), the mean square displacement in lateral direction. 

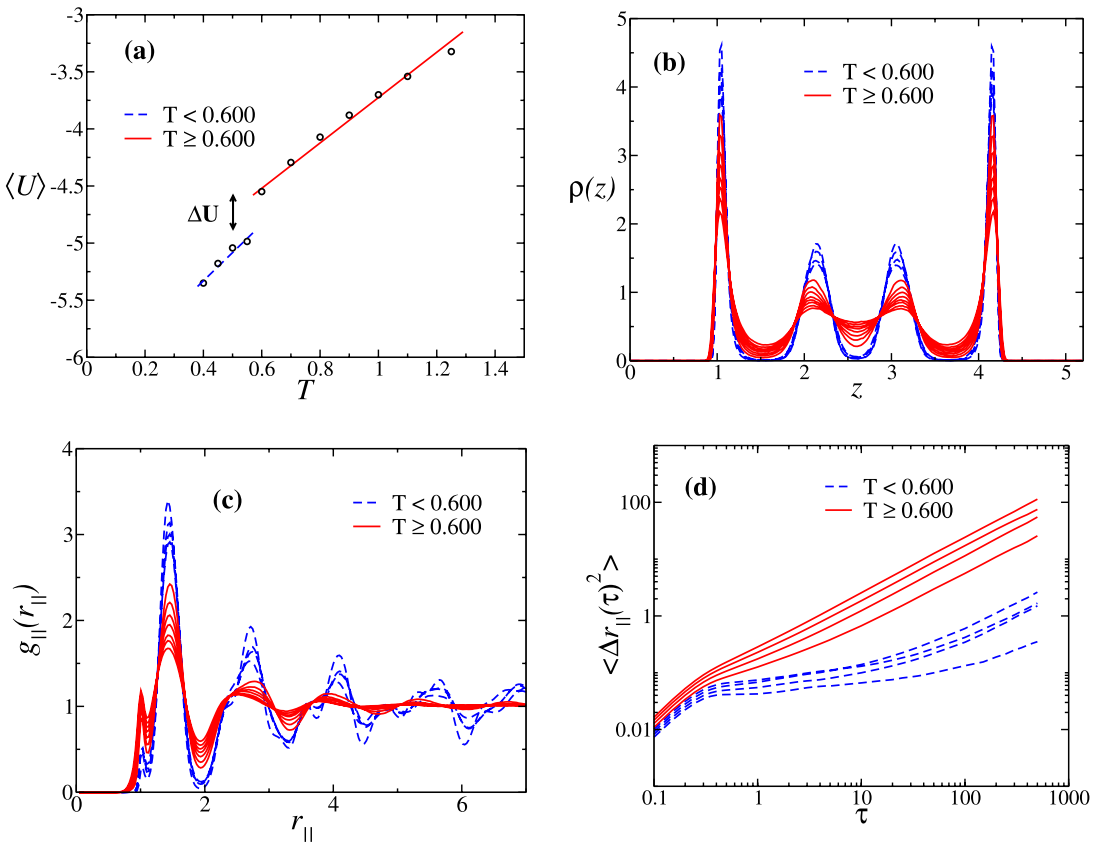

FIG. 7. System with plates separated at $d=5.2$ and density $\rho=0.536$. In (a), the mean potential energy as a function of temperature; in (b), the transversal density profile; in (c), the lateral radial distribution function $\left(g_{\|}\left(r_{\|}\right)\right)$for the contact layer; and in (d), the mean square displacement in lateral direction. layering allows for a high density interlayer making the full high density liquid only to appear at high densities.

For the plates separations $d=4.2$ and 5.2, the phase diagrams illustrated in Figs. 5(b) and 5(c) show the presence of a VLCP also shifted to lower temperatures when compared with the bulk system. However, the TMD line and the LLCP could not be determined.

Due to the increase of the entropic effects for a system under confinement, the melting line and the $L L C P$ should in principle move to lower temperatures. Whereas, we observed that the melting temperatures $\left(T_{m}\right)$ for the confined systems are higher than the bulk system. In addition, the change in the value of $T_{m}$ is not monotonic with $d$ similarly with what is observed in atomistic ${ }^{29}$ and waterlike fluids. ${ }^{41}$

In order to understand why the melting line moves to higher temperatures, covering the TMD and the $L L C P$, the structure in this region was analyzed. For this purpose, the transition is analyzed for $d=2.5$ and $d=5.2$. Fig. 6 in (a) illustrates the mean potential energy as a function of temperature, in (b) shows the transversal density profile, in (c) plots the lateral radial distribution function $\left(g_{\|}\left(r_{\|}\right)\right)$for the contact layer, and in (d) presents the mean square displacement in lateral direction for $d=2.5$ and $\rho=0.536$. We observe clearly a first order phase transition between an amorphous solid and a liquid phases. A discontinuous behavior was detected at
$T=0.50$. For $T<0.50$, the energies have lower values; the density profiles and the $g_{\|}\left(r_{\|}\right)$have a well defined structure; and the $\left\langle\Delta r^{2}(t)\right\rangle$ has a small inclination, showing a typical behavior of an amorphous solid phase. Whereas, for $T \geq 0.500$, the energy shows high values; and the density profiles, the $g_{\|}\left(r_{\|}\right)$, and the $\left\langle\Delta r^{2}(t)\right\rangle$ present a characteristic behavior of liquid phase. Amorphous solid-liquid first order phase transition was already observed for TIP5P model confined between smooth hydrophobic plates. ${ }^{65}$ The density profile shown in Figure 6(b), however, indicates that amorphous solid phase is not structured inside each layer but is present in the space between layers.

In the case of $d=5.2$ illustrated in Figure 7, the first order transition is observed at $T=0.06$ because at this temperature, the energy has a jump in (a), the radial distribution function shows a change in the structure from liquid to amorphous solid in (c), and the mean square displacement changes from nonzero to zero diffusion in (d). The density profile illustrated in Figure 7(b) differently than what is observed for $d=2.5$ shows that the amorphous solid structure is confined to a single layer.

The different ways in which the amorphous solid structures accommodate for the cases $d=2.5$ and $d=5.2$ under confinement explain the non-monotonic behavior of the melting temperature. While for strong confinement the amorphous solid forms across the layers in the region $d=5.2$ (and also $d=4.2$ ), the amorphous solid structure is confined to a single
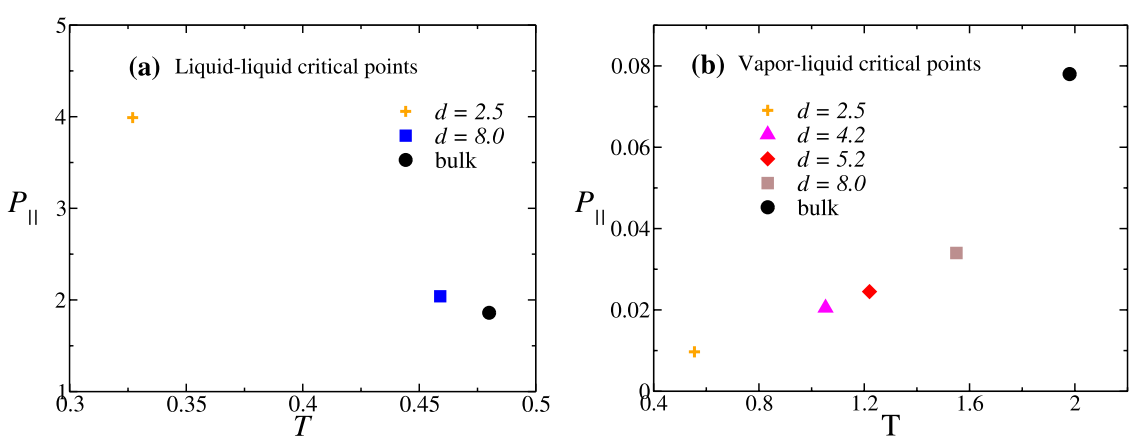

FIG. 8. Location of (a) $L L C P$ and (b) $V L C P$ for all the distances between the smooth plates. 

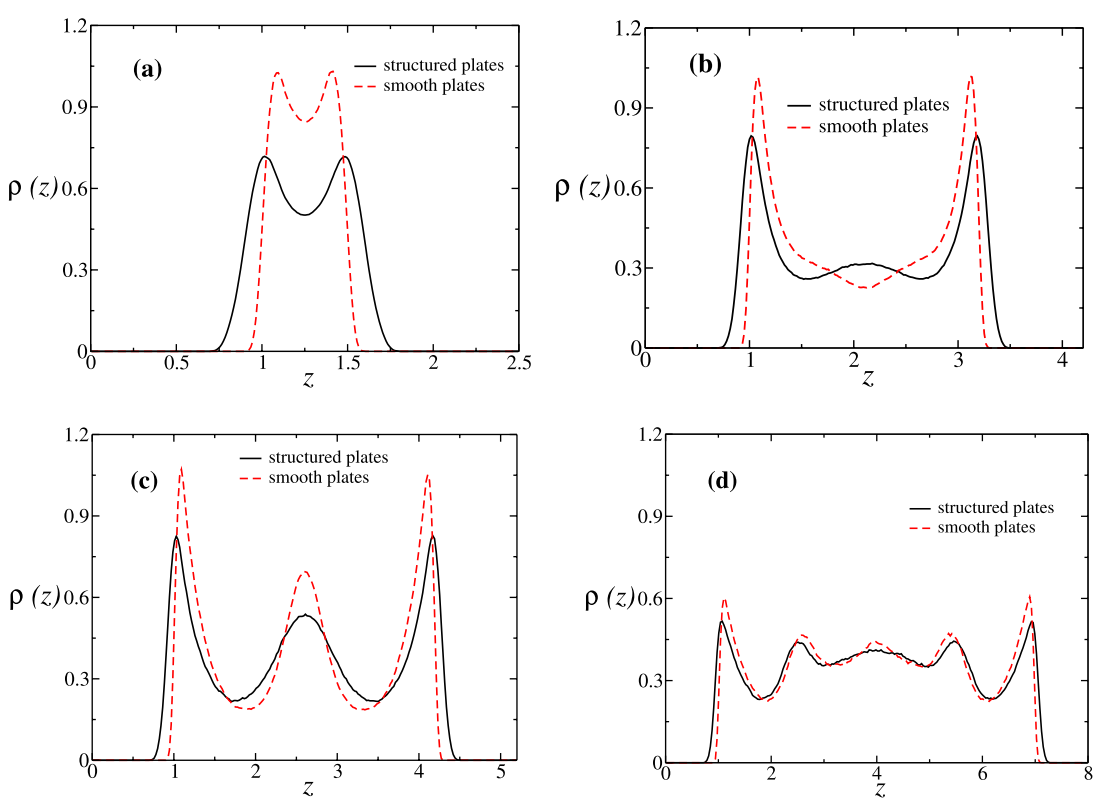

FIG. 9. Comparison of transversal density profile for systems confined by structured and smooth plates at $T$ $=0.80$ and different densities at (a) $d$ $=2.5$, (b) $d=5.2$, and (c) $d=8.0$. The confinement at $d=4.2$ is not shown for simplicity. layer. As the distance increases further the amorphous solid is again formed across layers approaching the bulk structure.

The effects of the confinement in the critical points of water are dependent on the geometry and wall structure of confinement that are being considered. For example, when the water is confined in the pore matrix, ${ }^{64}$ the $L L C P$ and the TMD line are shifted to lower temperatures and higher pressures in relation to bulk. But, in aqueous solutions of $\mathrm{NaCl}$, Corradini and Gallo ${ }^{66}$ show that the increase of salt concentration in water (TIP4P) shifts the $L L C P$ to higher temperatures and lower pressures in relation to bulk. Our results for the $L L C P$ and $V L C P$ are summarized in Fig. 8 and are in good agreement with the results for the pore media. ${ }^{64}$ This suggests that the salt/water long-range order interaction leads to changes in the water phase behavior what is not present in the short-range wall-particle interaction modeled by our system.
Experimental results show a non-monotonic behavior for the melting line and a strong dependence with the quality of the nanopore walls ${ }^{29}$ what is observed in our results. In Sec. III B, we will exam how the structure of the plates also has an important effect in the solidification of the system and in the location of the anomalies and critical points.

\section{B. Structured plates}

The second scenario we address here is the effect of the structure in the wall has in the thermodynamic and dynamical behaviors of the confined liquid. In this case, the plates are constructed by spherical particles in a square lattice, as sketched in Fig. 1(b). The interaction potential between fluid particles and walls particles is given by the WCA potential (Eq. (4)). A layering structure similar to picture observed for smooth plates
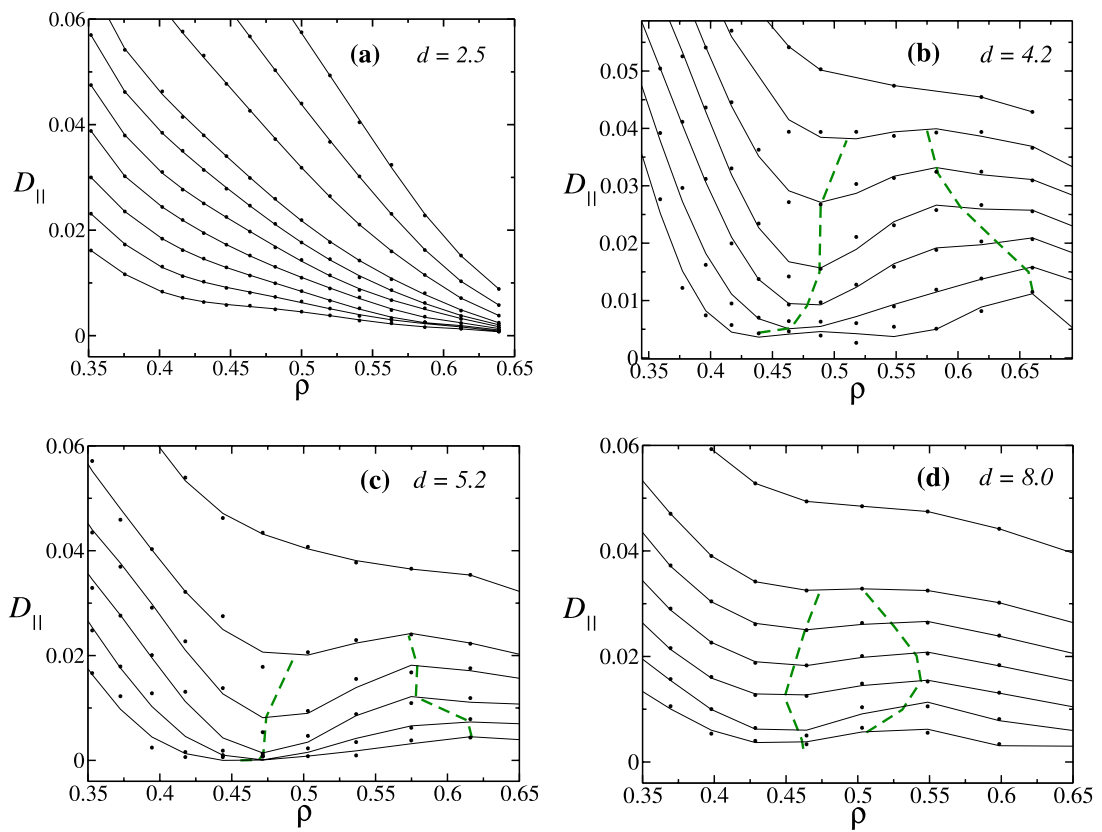

FIG. 10. Diffusion coefficient as a function of density for (a) $d=2.5$ and isotherms $0.50,0.55,0.60,0.65,0.70$, $0.75,0.80,0.90,1.00$, and 1.10 ; (b) $d=4.2$ and isotherms $0.60,0.65,0.70$, $0.75,0.80,0.85$, and 0.90 ; (c) $d=5.2$ and isotherms $0.50,0.55,0.60,0.65$, 0.70 , and 0.80 ; and (d) $d=8.0$ and isotherms $0.45,0.50,0.55,0.60,0.65$, 0.70 , and 0.80 . The dots are the simulated data and the black solid lines are polynomial fits. The dashed green lines bound the region where the diffusions are anomalous. 

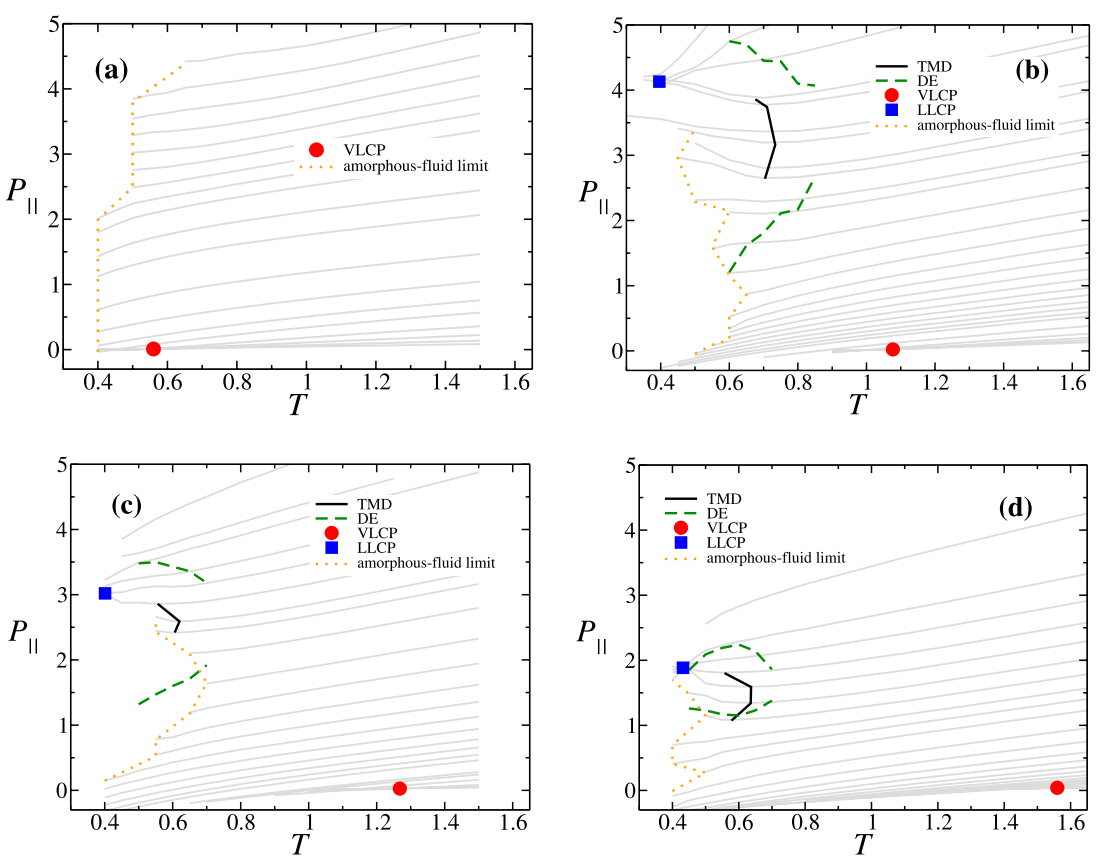

FIG. 11. Parallel pressure versus temperature phase diagram for systems with structured plates separated by distances (a) $d=2.5$, (b) $d=4.2$, (c) $d$ $=5.2$, and (d) $d=8.0$. The thin lines represent different isochores. analyzed in Sec. III A is also present for structured plates. In Fig. 9, the transversal density profiles for smooth and structured plates are compared for (a) for $d=2.5$ and $\rho=0.310$, (b) for $d=5.2$ and $\rho=0.334$, and (c) for $d=8.0$ and $\rho=0.321$. In all these cases, the temperature is the same, $T=0.80$. As the nanopore width decreases, the difference in the layer structure between the smooth and the structured walls increases. For $d=8.0$, the fluid exhibits almost the same density profile for the two types of confinement. This shows that for confined systems the fluid density profile is affected by the nanopore structure, particularly for strongly confined systems.

Another property of the liquid in which the structure of the confining surface might matter is the diffusion. Fig. 10 illustrates the diffusion coefficient in the parallel direction to the plates as a function of the fluid density for nanopores with size $d=2.5, d=4.2, d=5.2$, and $d=8.0$. The diffusion anomaly was observed for systems with plates separated at $d=4.2, d=5.2$, and $d=8.0$, while for $d=2.5$, no anomalous behavior was detected in the range of temperatures studied-at low temperatures, the fluid presents solidification, lead mainly by the nanopore structure.

Comparing the dynamical behavior of the systems, we verify that the fluid confined between structured plates behaves completely different from the smooth cases, particularly for small values of $d$. For $d=2.5$, systems confined by smooth plates show a large region of pressures and temperatures in which the diffusion anomaly is present (Fig. 4), while for structured walls, the fluid dynamically behaves like normal systems, without diffusion anomaly for the range of temperatures studied. The reason for this difference is that the structure of the wall plays a very important role in the structure of the liquid close to the wall, and since at $d=2.5$ the liquid is closer to the wall when compared with the smooth plates, the structure the wall determines the arrangement of the liquid. The liquid particles will be able to occupy the space between the wall particles.

The parallel pressure versus temperature phase diagrams are shown in the Fig. 11 for (a) $d=2.5$, (b) $d=5.2$, (c) $d=4.2$, and (d) $d=8.0$. The lines in the graph go as follows: the $T M D$ lines for each case are represented by solid lines, the diffusion extremes by dashed lines, the $V L C P$ by squares, the $L L C P$ by circles, and the limit between fluid and amorphous solid phases by dotted lines.

For structured nanopores with $d=2.5$, the density and diffusion anomalies and the $L L C P$ are not observed outside the amorphous regions. This is an effect of the influence of the wall-water potential that favors particles close to the wall to occupy the spaces between wall particles. Then the particle-particle two length scales competition that leads to the presence of density and diffusion anomalies does not happen, instead there is a competition between particle-particle and wall-particle interactions. The solidification for the system
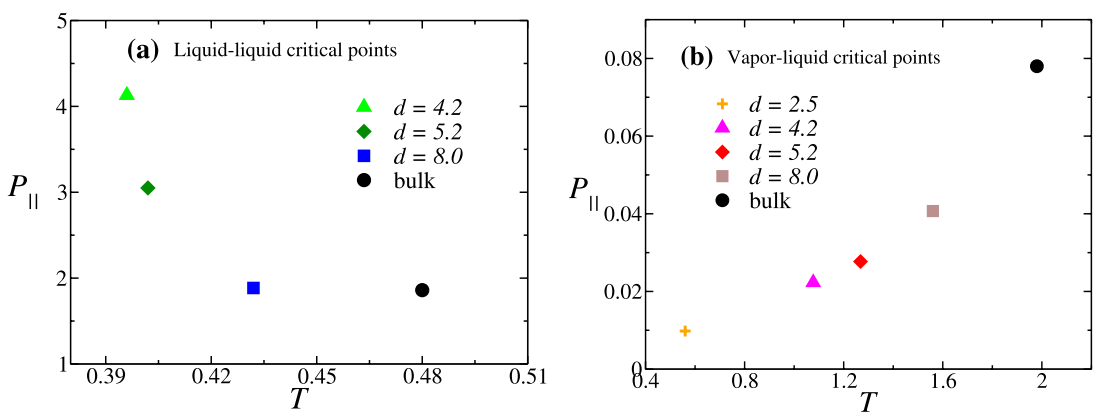

FIG. 12. Location of (a) $L L C P$ and (b) $V L C P$ for all the distances between the structured plates. 
in this case is similar to what happens in Sec. III A for $d$ $=4.2, d=5.2$, and $d=8$. In this case, however, the melting temperatures are lower than in the smooth potential case. The competition between the wall-particle interaction that favors one solid arrangement with the particle-particle interaction that favors other arrangement explains the difference between the melting for rough and smooth walls. Classical water model TIP5P confined between structured hydrophobic plates also presents a shift for higher temperatures. ${ }^{67}$

Fig. 12 summarizes the behavior of (a) $L L C P$ and (b) $V L C P$ for the different nanopores sizes and structured walls. The location of both critical points changes with the distances between the plates. As the nanopore width $d$ decreases, the $L L C P$ goes to lower temperatures and higher pressures, while the $V L C P$ is shifted to lower temperatures and lower pressures too.

\section{CONCLUSIONS}

In this work, we have studied the effects of the nanopore structure and of the water potential length scales in the waterlike properties of an anomalous fluid. First, we tested the effect of using a three length scales potential for analyzing the fluid behavior. In this case, the system confined by very small distances exhibits a different behavior when compared with confinement by intermediate and large distances. This difference can be explained by the arrangement of the fluid particles in the first, second, or third length scale of the potential. Then we check the differences in the thermodynamic and dynamic anomalies of the fluid when it was confined between smooth and structured walls. When observed, the density and diffusion anomalies are shifted to lower temperatures and higher pressures in relation to bulk for both kinds of confinement. However, the critical points and the limit between solid and fluid phases present a significant difference for each system. For high degrees of confinement, the properties of the fluid are very well defined when confined by smooth nanopores, but the fluid crystallizes for structured walls and small $d$. For intermediates separation of walls, smooth confinement presents solidification, and structured confinement do not. So, a non-monotonic behavior is observed in the properties of the fluid with $d$ when confined by smooth plates and a monotonic behavior with $d$ when confined between structured plates. The scales of the fluid-fluid and the fluid-plate interaction potential are responsible for the different behaviors observed for each kind of confinement.

\section{ACKNOWLEDGMENTS}

We thank for financial support the Brazilian science agencies, $\mathrm{CNPq}$ and Capes. This work is partially supported by CNPq, INCT-FCx.

\footnotetext{
${ }^{1}$ M. Chaplin, Seventy two anomalous properties of water, see http://www1. 1sbu.ac.uk/water/water_anomalies.html (2015).

${ }^{2}$ R. Waler, Essays of Natural Experiments (Johnson Reprint, New York, 1964).

${ }^{3}$ G. S. Kell, J. Chem. Eng. Data 20, 97 (1975).
}

${ }^{4}$ C. A. Angell, E. D. Finch, L. A. Woolf, and P. Bach, J. Chem. Phys. 65, 3063 (1976).

${ }^{5}$ P. H. Poole, F. Sciortino, U. Essmann, and H. E. Stanley, Nature 360, 324 (1992).

${ }^{6}$ Y. Liu, J. C. Palmer, A. Z. Panagiotopoulos, and P. G. Debenedetti, J. Chem. Phys. 137, 214505 (2012).

${ }^{7}$ J. C. Palmer et al., Nature 510, 385-388 (2014).

${ }^{8}$ A. Taschin, P. Bartolini, R. Eramo, E. Righini, and R. Torre, Nat. commun. 4, 2401 (2013).

${ }^{9}$ I. Brovchenko, A. Geiger, and A. Oleinikova, J. Chem. Phys. 123, 044515 (2005).

${ }^{10}$ P. H. Poole, S. R. Becker, F. Sciortino, and F. W. Starr, J. Phys. Chem. B 115 , 14176 (2011).

${ }^{11}$ P. H. Poole, R. K. Bowles, I. Saika-Voivod, and F. Sciortino, J. Chem. Phys. 138, 034505 (2013).

${ }^{12}$ A. Kesselring et al., J. Chem. Phys. 138, 244506 (2013).

${ }^{13}$ F. W. Starr, Nat. Phys. 10, 628 (2014).

${ }^{14}$ T. Yagasaki, M. Matsumoto, and H. Tanaka, Phys. Rev. Lett. 89, 020301(R) (2014).

${ }^{15}$ T. Morishite, Phys. Rev. Lett. 87, 105701 (2001).

${ }^{16}$ I. Saika-Voivod, F. Sciortino, and P. Poole, Phys. Rev. E 63, 011202 (2001).

${ }^{17}$ E. Lascaris, M. Hemmati, S. V. Buldyrev, H. E. Stanley, and C. A. Angell, J. Chem. Phys. 140, 224502 (2014).

${ }^{18}$ V. V. Vasisht, S. Saw, and S. Sastry, Nat. Phys. 7, 549 (2011).

${ }^{19}$ J. N. Glosli and F. H. Ree, Phys. Rev. Lett. 82, 4659 (1999).

${ }^{20}$ S. Scandolo, Proc. Natl Acad. Sci. U. S. A. 100, 3051 (2003).

${ }^{21}$ F. Smallenburg, L. Filion, and F. Sciortino, Nature Phys. 10, 653 (2014).

${ }^{22}$ D. T. Limmer and D. Chandler, J. Chem. Phys. 135, 135503 (2011).

${ }^{23}$ D. T. Limmer and D. Chandler, J. Chem. Phys. 138, 214504 (2013).

${ }^{24}$ C. A. Angell, J. Non-Cryst. Solids 407, 246 (2015).

${ }^{25}$ L. Liu, S.-H. Chen, A. Faraone, S.-W. Yen, and C.-Y. Mou, Phys. Rev. Lett. 95, 117802 (2005)

${ }^{26}$ A. Nagoe, S. Iwaki, M. Oguni, and K. Tôzaki, J. Phys. Soc. Jpn. 83, 094601 (2014).

${ }^{27}$ F. Mallamace et al., J. Chem. Phys. 141, 18 C504 (2014).

${ }^{28}$ S. Jähnert et al., Phys. Chem. Chem. Phys. 10, 6039 (2008).

${ }^{29}$ J. Deschamps, F. Audonnet, N. Brodie-Linder, M. Schoeffel, and C. AlbaSimionesco, Phys. Chem. Chem. Phys. 12, 1440 (2010).

${ }^{30}$ M. Erko, N. Cade, A. G. Michette, G. H. Findenegg, and O. Paris, Phys. Rev. B 84, 104205 (2011).

${ }^{31}$ K. Morishige and K. Kawano, J. Chem. Phys. 110, 4867 (1999).

${ }^{32}$ D. W. Hwang, C.-C. Chu, A. K. Sinha, and L.-P. Hwang, J. Chem. Phys. 126, 044702 (2007).

${ }^{33}$ S. Kittaka, K. Sou, T. Yamaguchi, and K. Tozaki, Phys. Chem. Chem. Phys 11, 8538 (2009).

${ }^{34}$ A. Faraone, K.-H. Liu, C.-Y. Mou, Y. Zhang, and S.-H. Chen, J. Chem. Phys. 130, 134512 (2009).

${ }^{35}$ P. C. Hemmer and G. Stell, Phys. Rev. Lett. 24, 1284 (1970).

${ }^{36}$ E. A. Jagla, Phys. Rev. E 58, 1478 (1998).

${ }^{37}$ L. Xu et al., Proc. Natl. Acad. Sci. U. S. A. 102, 16558 (2005).

${ }^{38}$ A. B. de Oliveira, P. A. Netz, T. Colla, and M. C. Barbosa, J. Chem. Phys. 124, 084505 (2006).

${ }^{39}$ E. Lomba, N. G. Almarza, C. Martin, and C. McBride, J. Chem. Phys. 126, 244510 (2007).

${ }^{40}$ J. da Silva, E. Salcedo, A. B. Oliveira, and M. C. Barbosa, J. Phys. Chem. 133, 244506 (2010).

${ }^{41}$ L. Krott and M. C. Barbosa, J. Chem. Phys. 138, 084505 (2013).

${ }^{42}$ L. B. Krott and M. C. Barbosa, Phys. Rev. E 89, 012110 (2014).

${ }^{43}$ L. B. Krott and J. R. Bordin, J. Chem. Phys. 139, 154502 (2013).

${ }^{44}$ J. R. Bordin, L. B. Krott, and M. C. Barbosa, J. Phys. Chem. C 118, 9497 (2014).

${ }^{45}$ J. R. Bordin, L. B. Krott, and M. C. Barbosa, J. Chem. Phys. 141, 144502 (2014).

${ }^{46}$ L. B. Krott, J. R. Bordin, and M. C. Barbosa, J. Phys. Chem. B 119, 291 (2015).

${ }^{47}$ J. R. Bordin, A. B. de Oliveira, A. Diehl, and M. C. Barbosa, J. Chem. Phys 137, 084504 (2012).

${ }^{48}$ J. R. Bordin, A. Diehl, and M. C. Barbosa, J. Phys. Chem. B 117, 7047 (2013).

${ }^{49}$ J. R. Bordin, J. S. Andrade, Jr., A. Diehl, and M. C. Barbosa, J. Chem. Phys. 140, 194504 (2014).

${ }^{50}$ H. Holzmann, R. Ludwig, A. Geiger, and D. Paschek, Angew. Chem., Int. Ed. 46, 8907 (2007). 
${ }^{51}$ O. Mishima, J. Chem. Phys. 133, 144503 (2010).

${ }^{52}$ W. P. Krekelberg, V. K. Shen, J. R. Errington, and T. M. Truskett, J. Chem. Phys. 135, 154502 (2011).

${ }^{53}$ K. Yang, Y. Lin, X. Lu, and A. V. Neimark, J. Colloid Interface Sci. 362, 382 (2011).

${ }^{54} \mathrm{P}$. Allen and D. J. Tildesley, Computer Simulation of Liquids (Oxford University Press, Oxford, 1987).

${ }^{55}$ N. Barraz, Jr., E. Salcedo, and M. Barbosa, J. Chem. Phys. 131, 094504 (2009).

${ }^{56}$ W. G. Hoover, Phys. Rev. A 31, 1695 (1985)

${ }^{57}$ W. G. Hoover, Phys. Rev. A 34, 2499 (1986).

${ }^{58}$ T. Head-Gordon and F. H. Stillinger, J. Chem. Phys. 98, 3313 (1993).
${ }^{59}$ P. Kumar, S. V. Buldyrev, F. W. Starr, N. Giovambattista, and H. E. Stanley, Phys. Rev. E 72, 051503 (2005).

${ }^{60}$ M. Meyer and H. E. Stanley, J. Phys. Chem. B 103, 9728 (1999).

${ }^{61}$ N. Giovambattista, P. J. Rossky, and P. G. Debenedetti, J. Phys. Chem. B 113, 13723 (2009)

${ }^{62}$ E. B. Moore, J. T. Allen, and V. Molinero, J. Phys. Chem. C 116, 7507 (2012).

${ }^{63}$ T. M. Truskett, P. G. Debenedetti, and S. Torquato, J. Chem. Phys. 114, 2401 (2001).

${ }^{64}$ E. G. Strekalova et al., J. Biol. Phys. 38, 97 (2012).

${ }^{65}$ S. Han, M. Y. Choi, P. Kumar, and H. E. Stanley, Nat. Phys. 6, 685 (2010).

${ }^{66}$ D. Corradini and P. Gallo, J. Phys. Chem. B 115, 14161 (2011).

${ }^{67}$ R. Zangi, J. Phys.: Condens. Matter 16, S5371 (2004). 